\title{
Guided design of copper oxysulfide superconductors
}

\author{
Chuck-Hou Yee,* Turan Birol, and Gabriel Kotliar \\ Dept. of Physics 8 Astronomy, Rutgers, The State University of New Jersey, Piscataway, NJ 08854, USA
}

(Dated: October 8, 2018)

\begin{abstract}
We describe a framework for designing novel materials, combining modern first-principles electronic structure tools, materials databases, and evolutionary algorithms capable of exploring large configurational spaces. Guided by the chemical principles introduced by Antipov, et. al., for the design and synthesis of the $\mathrm{Hg}$-based high-temperature superconductors, we apply our framework to design a new layered copper oxysulfide, $\mathrm{Hg}(\mathrm{CaS})_{2} \mathrm{CuO}_{2}$. We evaluate the prospects of superconductivity in this oxysulfide using theories based on charge-transfer energies, orbital distillation and uniaxial strain.
\end{abstract}

The superconductors with the highest known transition temperatures at ambient pressure are all layered compounds containing planes of copper and oxygen where the superconducting electrons reside, separated by "spacer layers" composed of other elements. For a given compound, varying the doping level to an optimal value near 0.15 holes per copper maximizes the superconducting transition temperature $T_{\mathrm{c}}$. Since the copper oxide planes are a common ingredient, the large variabilty in optimal $T_{\mathrm{c}}$ 's between compounds must then be controlled by the spacer layers, which function to tune the chemical and structural properties of the copper oxide layer. Finding novel compositions for the spacer layers is key to discovering new superconductors with higher transition temperatures.

Theoretical design of new compounds is challenging due to the vast combinatorial space of elements and the large number of constraints: preferred oxidation state, electronegativity, atomic radii, preferred local coordination environment, and overall electrical neutrality. These microscopic properties in turn determine the local structural stability, global configurational minimum, and thermodynamic stability. Finding the global low-energy structure is the computational bottleneck. If a given composition results in a stable compound, we still need to determine whether it tunes the low-energy Hamiltonian so that $T_{\mathrm{c}}$ is enhanced, which imposes further screening criteria.

Rather than design a compound from scratch, we adopt a more moderate approach: we take as a starting point the family of cuprates with the highest transition temperatures, the $\mathrm{Hg}$-based cuprates, and modulate its spacer layers. We benefit from the vast body of chemical intuition accumulated for the cuprates, for instance, laid out especially clearly in Ref. 1, and use modern electronic structure methods [2,3], materials databases $[4,5]$ and evolutionary algorithms [6] to efficiently screen for compositions which have the desired structure and the best prospects for stability. We use three proposed theories, based on epitaxial strain [7], the charge-transfer energy [8], and orbital distillation [9], to evaluate the resultant compound's prospects for superconducivity. In this manuscript, we describe the general framework for guided design of new materials, and demonstrate its workflow by applying the principles to design a new layered copper oxysulfide $\mathrm{Hg}(\mathrm{CaS})_{2} \mathrm{CuO}_{2}$, which we abbreviate (HC$\mathrm{SCO}$ ). If it can be synthesized and doped, we believe HCSCO will be a high-temperature superconductor.

\section{RATIONAL DESIGN}

The cuprates are a functional stack: the composition of each layer is chosen to play a specific role (Fig. 1). The central copper oxide $\left(\mathrm{CuO}_{2}\right)$ plane supports superconductivity and roughly constrains the in-plane lattice constant. The remaining layers must tune the chemical potential of the $\mathrm{CuO}_{2}$ layer without rumpling the plane or introducing disorder, and isolate each $\mathrm{CuO}_{2}$ plane to create a $2 \mathrm{D}$ system.

In the $\mathrm{Hg}$-cuprates, the $\mathrm{HgO}_{\delta}$ layer harbors dopant atoms which tune the chemical potential. The $\mathrm{BaO}$ layers immediately adjacent to the $\mathrm{CuO}_{2}$ plane spatially separate the superconducting electrons from the detrimental effects of the disordered dopant layer [10,11]. Additionally, the highly ionic nature of the $\mathrm{BaO}$ layer means they do not capture dopant electrons intended for the $\mathrm{CuO}_{2}$ plane. The preference of $\mathrm{Hg}$ to be dumbbell coordinated bonds the entire structure together without introducing structural distortions [1]. Finally, the highly ionic O$\mathrm{Hg}$-O dumbbells minimize $c$-axis hopping to maintain 2dimensionality.

Due to their spatial proximity, the adjacent $\mathrm{BaO}$ layers tune the hoppings and interaction strengths of the in-plane Hamiltonian. Designing compounds with novel adjacent layers provides a mechanism for controlling superconductivity by, e.g., reducing the charge-transfer energy. We quickly realized the most stringent constraint is structural stability, so we focused first on isolating stable candidates, then subsequently investigating their electronic properties.

To maximize the likelihood that a proposed composition is stable, we note that the layers adjacent to the $\mathrm{CuO}_{2}$ plane form a rock salt structure. Using materials databases, we selected all naturally occurring rock salt compounds AX, composed of an cation A and an an- 


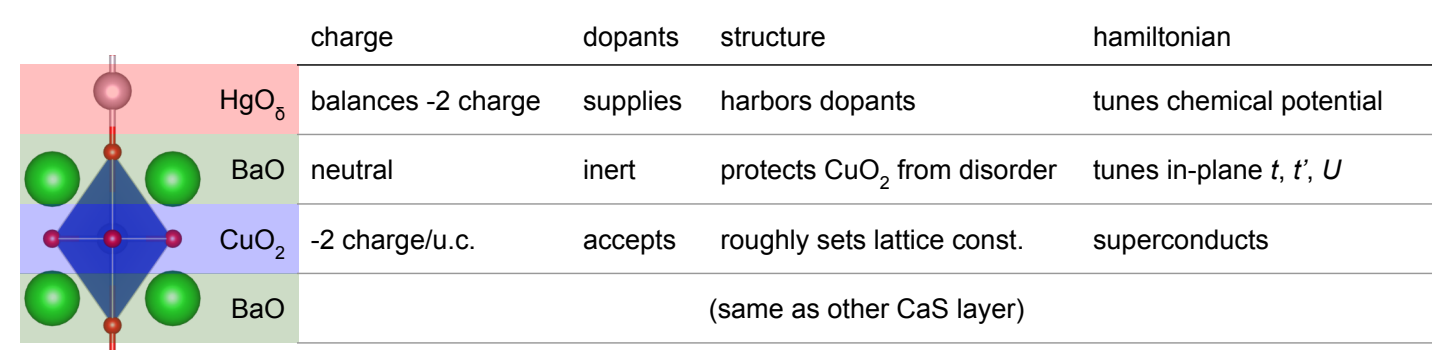

FIG. 1. Each layer in the structural stack of $\mathrm{HgBa}_{2} \mathrm{CuO}_{4+\delta}$ performs a specific chemical, structural and electronic function. We focused on tuning the layers immediately adjacent to the $\mathrm{CuO}_{2}$ plane which function to tune the in-plane hamiltonian, but must also be charge neutral and not interfere with doping.

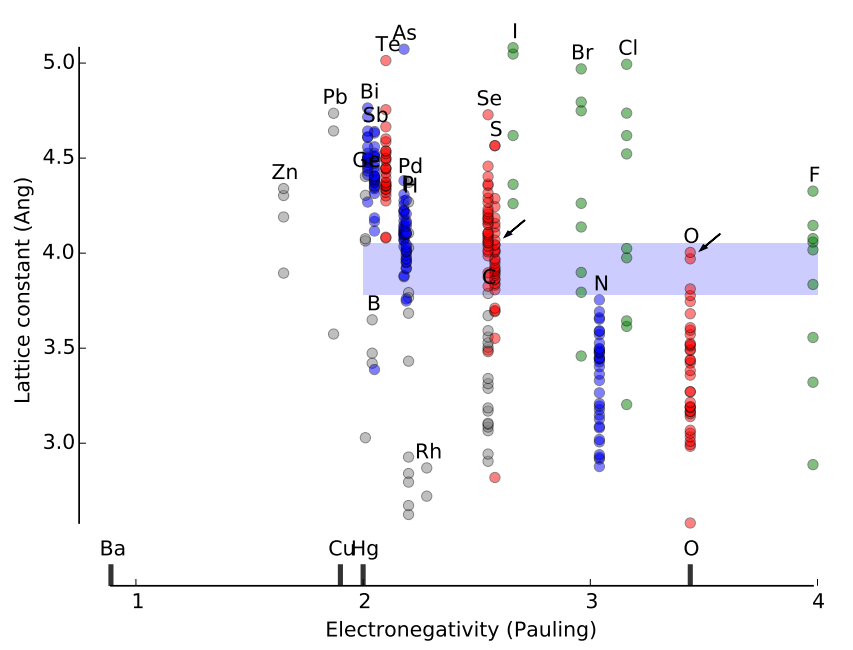

FIG. 2. Rock salt compositions AX plotted as a function of their lattice constant vs. electronegativity of the anion X, and color-coded by anion's nominal valence. Electronegativities of elements existing in $\mathrm{HgBa}_{2} \mathrm{CuO}_{4}$ are marked. We seek compositions which have (a) lattice constants comparable to the in-plane $\mathrm{Cu}-\mathrm{Cu}$ distance in the cuprates $(\sim 3.80-3.95 \AA)$, and (b) anion electronegativities greater than that of $\mathrm{Cu}$, as dopants intended for the copper oxide plane would otherwise be captured by the anion, resulting in additional Fermi surfaces. The compositions of interest are marked by a shaded rectangle. Arrows point to $\mathrm{BaO}$ and $\mathrm{CaS}$.

ion $\mathrm{X}$ (Fig. 2). The phase space is large and the rate limiting step is structural prediction, so we quickly prescreen candidates by discarding compositions with (1) large lattice mismatches relative to the in-plane $\mathrm{Cu}-\mathrm{Cu}$ distance, which we took to be $3.82 \AA$, and (2) anions less electronegative than $\mathrm{Cu}$, as these anions would capture dopants intended for the superconducting plane, producing additional Fermi surfaces.

Given the remaining list of potential compositions, we screened for stable compounds as follows:

1. Local stability - substitute $\mathrm{AX}$ for $\mathrm{BaO}$ layer of $\mathrm{HBCO}$ structure forming $\mathrm{Hg}(\mathrm{AX})_{2} \mathrm{CuO}_{2}$, fully relax the structure, then compute the phonons. Dis- card if there are unstable phonons.

2. Configurational minimum - place one formula unit of the elements $(1 \mathrm{Hg}, 2 \mathrm{~A}, 2 \mathrm{X}, 1 \mathrm{Cu} 2 \mathrm{O})$ in a box and apply evolutionary algorithms to find most energetically stable crystal structure.

3. Thermodynamic stability - compute convex hull of $\mathrm{Hg}-\mathrm{A}-\mathrm{X}-\mathrm{Cu}-\mathrm{O}$ system and determine whether $\mathrm{Hg}(\mathrm{AX})_{2} \mathrm{CuO}_{2}$ lies below the hull. Synthesis generally proceeds in oxygen environments so the hull is computed with fixed $\mathrm{Hg}-\mathrm{A}-\mathrm{X}-\mathrm{Cu}$ stoichiometry and varying oxygen concentration, parameterized by the chemical potential $\mu\left(\mathrm{O}_{2}\right)$.

Compositions which pass all these hurdles have a good chance of being synthesizble in bulk. However, these criteria aren't rigid, as compositions which exhibit only weak phonon instabilities are likely synthesizable and possibly superconducting. Additionally, compositions that lie above the convex hull may also be synthesized via epitaxy or under high pressures.

\section{METHODS}

The pymatgen implementation of the Materials API [12] was used to access the Materials Project [4] database to select all structures with lattice constants within $\sim 5 \%$ of $3.9 \AA$, the nominal $\mathrm{Cu}-\mathrm{Cu}$ distance.

Structural relaxations within DFT were performed with VASP [2,3] using PAW potentials [13] and the PBEsol functional [14]. We computed phonons at the $(0,0),(\pi, 0)$ and $(\pi, \pi) k$-points using $1 \times 2$ and $\sqrt{2} \times \sqrt{2}$ supercells constructed with the help of ISOTROPY [15] to check local stability.

We performed a fixed-composition evolutionary search for crystal structure using USPEX [16, 17], with VASP as the underlying DFT engine. A unit cell consisting of one formula unit and an initial population of 256 random structures were used, decreasing to 64 in following generations. USPEX successfully found existing cuprate structures (LSCO, YBCO, HBCO) in benchmark tests, 
demonstrating its efficacy in the few-atom (8 atoms/f.u.) but many-species (5 elements) regime.

The Gibbs phase diagrams for the $\mathrm{Hg}-\mathrm{A}-\mathrm{X}-\mathrm{Cu}$ system at varying oxygen potentials $\mu\left(\mathrm{O}_{2}\right)$ were constructed using the phase diagram implementation in pymatgen [18]. We cross-checked these results with the PBE functional, which is known to give improved atomization energies [14], finding no significant differences in the relative energies between compounds.

Bandstructure orbital character was analyzed with WIEN2k [19] and structures plotted using VESTA [20]. Electronic parameters were extracted by downfolding to atomic-like orbitals, defined in [21].

\section{RESULTS}

Our materials design framework efficiently isolates compositions with desired crystal structures and properties. From roughly 350 rock salt compounds, prescreening based on lattice constants and electronegativity selected $\sim 20$ compositions. Screening for local stability via phonon modes left three compositions: CaS, ZrAs, and YbS. Configurational stability using evolutionary structure prediction showed only a single composition, $\mathrm{Hg}(\mathrm{CaS})_{2} \mathrm{CuO}_{2}$, adopted the $\mathrm{HBCO}$ structure. This compound has sulfur instead of oxygen as the apical atom and has the smaller calcium ion instead barium in the adjacent layer. We also performed structure prediction with two formula units, finding the same HBCO structure. Thermodynamic calculations show HCSCO is marginally unstable, lying $240 \mathrm{meV} /$ atom above the convex hull at $\mu\left(\mathrm{O}_{2}\right)=-16.48 \mathrm{eV}$, with an expected decomposition into $\mathrm{Hg}, \mathrm{Cu}, \mathrm{CaS}$, and oxygen gas (Fig. 3).

Turning to electronic structure (Fig. 4), we observe a single band crossing the Fermi level with strong $d_{x^{2}-y^{2}}$ character, similar to other single-layer cuprates. The Fermi surface extremely 2-dimensional, and comparable to HBCO. Table I summarizes a comparison of the salient electronic parameters extracted using downfolding between HCSCO and the single-layer HBCO superconductor.

\begin{tabular}{ccccccccccc} 
& \multicolumn{3}{c}{ Structure $(\AA)$} & & \multicolumn{5}{c}{ Parameters $(\mathrm{eV})$} \\
\cline { 2 - 3 } \cline { 7 - 9 } & $a$ & $\mathrm{c}$ & $d_{\text {apical }}$ & & $\Delta_{p d}$ & $\Delta E_{\mathrm{z}}$ & $t_{p d}$ & $t_{p p}$ & $t_{p p}^{\prime}$ \\
\hline HCSCO & 3.833 & 10.054 & 2.746 & & 1.94 & 0.20 & 1.35 & 0.61 & 0.09 \\
HBCO & 3.879 & 9.516 & 2.789 & & 1.93 & 1.87 & 1.25 & 0.65 & 0.16
\end{tabular}

TABLE I. Comparison of structural and electronic parameters of $\mathrm{HgBa}_{2} \mathrm{CuO}_{4}$ and $\mathrm{Hg}(\mathrm{CaS})_{2} \mathrm{CuO}_{2}$.

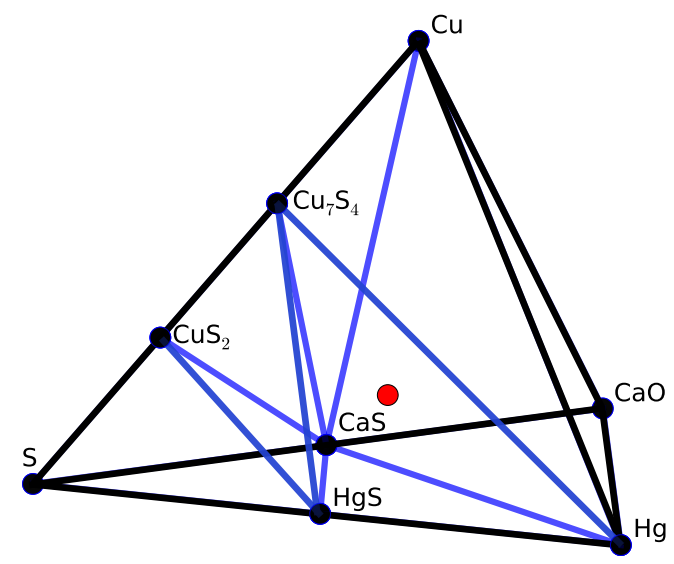

FIG. 3. The Gibbs phase diagram of the $\mathrm{Hg}-\mathrm{Ca}-\mathrm{S}-\mathrm{Cu}$ system at an oxygen chemical potential of $\mu\left(\mathrm{O}_{2}\right)=-16.48 \mathrm{eV}$, chosen because $\mathrm{Hg}(\mathrm{CaS})_{2} \mathrm{CuO}_{2}$ (red dot) is least unstable at this value. The phase diagram forms a tetrahedron with $\mathrm{S}, \mathrm{Hg}$, $\mathrm{Cu}$ and $\mathrm{CaO}$ at the vertices (elemental $\mathrm{Ca}$ isn't stable under this oxygen environment). HCSCO lies in the interior of the tetrahedron, on the triangular face formed by $\mathrm{Hg}, \mathrm{Cu}$ and CaS.

\section{DISCUSSION}

We evaluate the prospects of superconductivity in HCSCO based on three chemically-based proposals. Applying in-plane compression induces large increases in transition temperatures, with $d T_{\mathrm{c}} / d(\log a) \sim-600 \mathrm{~K}$ in LSCO [7] and $-4000 \mathrm{~K}$ in HBCO [1], where $a$ is the in-plane lattice constant. We are aware that many variables are involved, especially that compression may induce buckling, which decreases $T_{\mathrm{c}}$ [22]. However, assuming all other factors are held constant, the $1.6 \%$ compression of HCSCO relative to $\mathrm{HBCO}$ would cause a $60 \mathrm{~K}$ increase in $T_{\mathrm{c}}$.

An alternative proposal argues that tuning the chargetransfer energy $\Delta_{p d}$, which roughly corresponds to the effective Coulomb interaction, controls superconductivity [8]. As shown in Table I, the negligible difference in $\Delta_{p d}$ between HCSCO and HBCO, combined with similar hopping amplitudes, implies comparable $T_{\mathrm{c}}$ 's (Fig. 5). We expected that the increased polarizability of sulfur as compared to oxygen would reduce $\Delta_{p d}$, an effect which may be captured in methods beyond LDA, like the GW approximation.

Finally, the orbital distillation proposal argues that a large admixture of the apical orbitals, in particular the $\mathrm{Cu}-d_{z^{2}}$ orbital, into the $d_{x^{2}-y^{2}}$ band suppresses $T_{\mathrm{c}}$ [9]. Although the energy splitting $\Delta E_{z} \equiv E_{x^{2}-y^{2}}-E_{z^{2}}$ is quite small (Table I), there is no admixture of apical orbital charcter into the in-plane band (Fig. 4). Orbital distillation predicts $T_{\mathrm{c}}$ 's comparable to the $\mathrm{HBCO}$ superconductors.

Turning to stability against phase separation, our cal- 

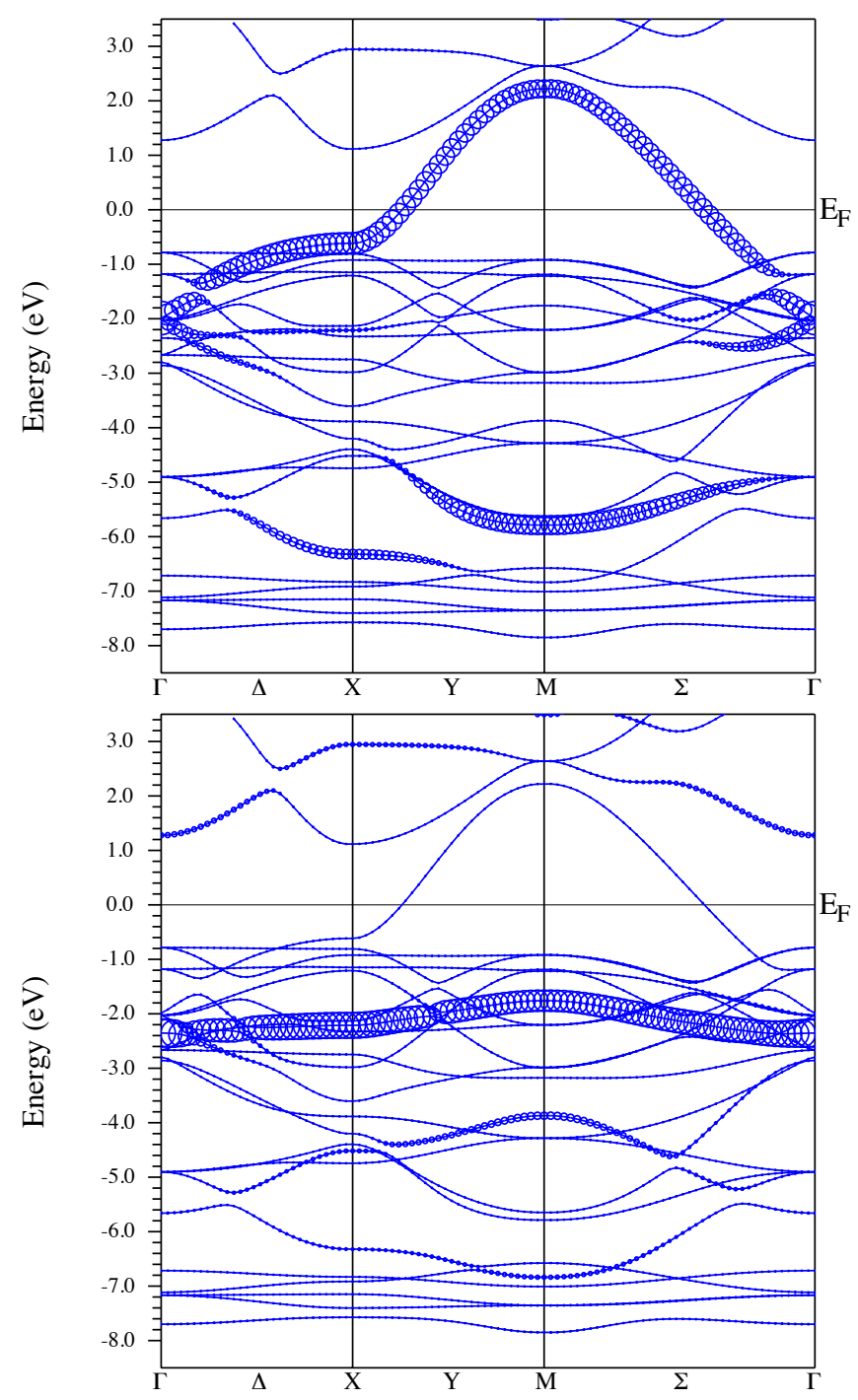

FIG. 4. Bandstructure of HCSCO, with $d_{x^{2}-y^{2}}$ (top) and $d_{z^{2}}$ (bottom) character highlighted. A single band crosses the Fermi level of mainly $d_{x^{2}-y^{2}}$ character with little admixture of $d_{z^{2}}$ character.

culations indicate HCSCO lies slightly above the convex hull, well within the systematic errors of the theory used to estimate total energies. Furthermore it is known that many interesting functional materials are metastable [25], protected from decay by large energetic barriers. In solid state synthesis, the Hg-cuprates are formed in the presence of gaseous $\mathrm{Hg}$ [26] in addition to $\mathrm{O}_{2}$, providing an additional tuning parameter which may enhance stability. Finally, prior experience with materials design indicates that compounds which are too stable may not be easily dopable $[27,28]$. These considerations suggest avenues for synthesis of HCSCO should be explored, both under pressure in bulk and especially via molecular beam epitaxy.

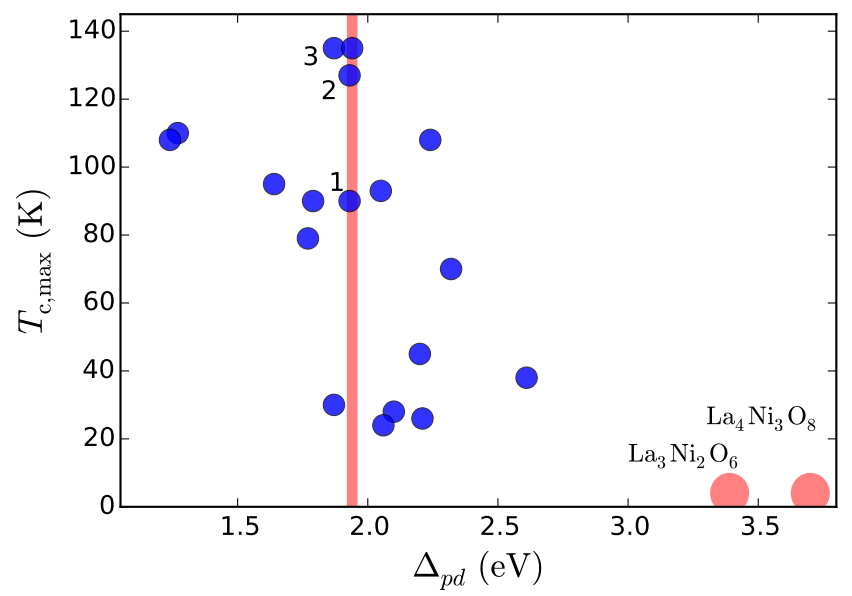

FIG. 5. Scatter-plot of the maximal superconducting transition temperature $T_{\mathrm{c}, \max }$ vs. charge-transfer energy $\Delta_{p d}$ for the copper oxides (see Ref. 8 for details). The vertical bar marks the $\Delta_{p d}$ of HCSCO, which is close to that of the other mercury cuprates (labeled 1, 2 and 3 by the number of $\mathrm{CuO}_{2}$ layers); thus, we expect similar transition temperatures. For comparison, we have also plotted the charge-transfer energies of two layered nickelates, $\mathrm{La}_{3} \mathrm{Ni}_{2} \mathrm{O}_{6}$ [23] and $\mathrm{La}_{4} \mathrm{Ni}_{3} \mathrm{O}_{8}$ [24], where the $\mathrm{Ni}$ valence can be tuned near $3 d^{9}$ and are predicted to be nonsuperconducting (red circles).

\section{CONCLUSIONS}

We have created a workflow for systematically designing novel compositions based on existing compound families. Guided by chemical intuition and materials databases, our workflow quickly isolates promising compositions for in-depth examination. Combining electronic structure tools, evolutionary algorithms, and methods for constructing phase diagram, we screen for local, configurational and thermodynamic stability to isolate new compounds with a high probability of synthesis.

We have applied this workflow to design a new layered copper oxysulfide, $\mathrm{Hg}(\mathrm{CaS})_{2} \mathrm{CuO}_{2}$, which we believe will be a high-temperature superconductor, with $T_{\mathrm{c}}$ 's comparable to those of the mercury cuprates. In the future, we expect the multi-layer family members of $\mathrm{HCSCO}$ will be designed, with formulas $\mathrm{Hg}(\mathrm{CaS})_{2} \mathrm{R}_{n-1}\left(\mathrm{CuO}_{2}\right)_{n}$ where $\mathrm{R}$ is a $2+$ cation placed between the $\mathrm{CuO}_{2}$ planes in the "infinite-layer" stack, as well as systematic extensions to the compositions of other cuprate families.

To date, cuprate superconductors exist with oxygen, chlorine and fluorine in the apical position. The synthesis HCSCO would produce the exciting prospect of adding a fourth, sulfur-based family. 


\section{ACKNOWLEDGEMENTS}

C.Y. and G.K. were supported by the DOE EFRC CES program. T.B. acknowledges the support of the Center for Materials Theory at Rutgers University.

* chuckyee@physics.rutgers.edu

[1] . Antipov and . Abakumov, Uspekhi Fizicheskih Nauk 178, 190 (2008).

[2] G. Kresse and J. Furthmller, Comput. Mat. Sci. 6, 15 (1996).

[3] G. Kresse and J. Furthmller, Phys. Rev. B 54, 11169 (1996).

[4] A. Jain, S. P. Ong, G. Hautier, W. Chen, W. D. Richards, S. Dacek, S. Cholia, D. Gunter, D. Skinner, G. Ceder, and K. a. Persson, APL Materials 1, 011002 (2013).

[5] A. Belsky, M. Hellenbrandt, V. L. Karen, and P. Luksch, Acta Crystallographica Section B Structural Science 58, 364 (2002).

[6] A. R. Oganov and C. W. Glass, The Journal of chemical physics 124, 244704 (2006).

[7] J. Locquet, J. Perret, J. Fompeyrine, and E. Mächler, Nature 394, 453 (1998).

[8] C. Weber, C. Yee, K. Haule, and G. Kotliar, EPL (Europhysics Letters) 100, 37001 (2012).

[9] H. Sakakibara, H. Usui, K. Kuroki, R. Arita, and H. Aoki, Physical Review Letters 105, 057003 (2010).

[10] K. Fujita, T. Noda, K. Kojima, H. Eisaki, and S. Uchida, Physical Review Letters 95, 097006 (2005).

[11] H. Hobou, S. Ishida, K. Fujita, M. Ishikado, K. Kojima, H. Eisaki, and S. Uchida, Physical Review B 79, 064507 (2009).

[12] S. P. Ong, W. D. Richards, A. Jain, G. Hautier, M. Kocher, S. Cholia, D. Gunter, V. L. Chevrier, K. a. Persson, and G. Ceder, Computational Materials Science 68, 314 (2013).

[13] P. E. Blöchl, Phys. Rev. B 50, 17953 (1994).
[14] J. P. Perdew, K. Burke, and M. Ernzerhof, Phys. Rev. Lett. 77, 3865 (1996).

[15] H. T. Stokes and D. M. Hatch, Journal of Applied Crystallography 38, 237 (2005).

[16] C. W. Glass, A. R. Oganov, and N. Hansen, Computer Physics Communications 175, 713 (2006).

[17] A. O. Lyakhov, A. R. Oganov, H. T. Stokes, and Q. Zhu, Computer Physics Communications 184, 1172 (2013).

[18] S. Ping Ong, L. Wang, B. Kang, and G. Ceder, Chemistry of Materials 20, 1798 (2008).

[19] P. Blaha, K. Schwarz, G. K. H. Madsen, D. Kvasnicka, and J. Luitz, WIEN2K, An Augmented Plane Wave + Local Orbitals Program for Calculating Crystal Properties (Karlheinz Schwarz, Techn. Universität Wien, Austria, 2001).

[20] K. Momma and F. Izumi, Journal of Applied Crystallography 41, 653 (2008).

[21] K. Haule, C.-H. Yee, and K. Kim, Physical Review B 81, 195107 (2010).

[22] W. Gao, Q. Liu, L. Yang, Y. Yu, F. Li, C. Jin, and S. Uchida, Physical Review B 80, 094523 (2009).

[23] V. V. Poltavets, K. A. Lokshin, S. Dikmen, M. Croft, T. Egami, and M. Greenblatt, Journal of the American Chemical Society 128, 9050 (2006), arXiv:arXiv:1103.2407v1.

[24] V. V. Poltavets, K. a. Lokshin, A. H. Nevidomskyy, M. Croft, T. a. Tyson, J. Hadermann, G. Van Tendeloo, T. Egami, G. Kotliar, N. Aproberts-Warren, A. P. Dioguardi, N. J. Curro, and M. Greenblatt, Physical Review Letters 104, 6 (2010), arXiv:1003.3276.

[25] X. Zhang, L. Yu, A. Zakutayev, and A. Zunger, Advanced Functional Materials 22, 1425 (2012).

[26] V. Alyoshin, D. Mikhailova, E. Rudnyi, and E. Antipov, Physica C: Superconductivity 383, 59 (2002).

[27] Z. P. Yin and G. Kotliar, EPL (Europhysics Letters) 101, 27002 (2013), arXiv:1301.7477.

[28] M. Retuerto, Z. Yin, T. J. Emge, P. W. Stephens, M.-r. Li, T. Sarkar, M. C. Croft, A. Ignatov, Z. Yuan, S. J. Zhang, C. Jin, R. Paria Sena, J. Hadermann, G. Kotliar, and M. Greenblatt, Inorganic Chemistry 54, 1066 (2015). 